\title{
Uma proposta para monitorar a poluição sonora através de smartphones e uma cidade virtual 3D
}

\author{
Frederico Severo Miranda ${ }^{1}$, Paulo Cézar Stadzisz ${ }^{1}$ \\ ${ }^{1}$ Universidade Tecnológica Federal do Paraná (UTFPR) \\ Avenida Sete de Setembro, 3165 - Curitiba - PR - Brasil \\ fdr.miranda@gmail.com, stadziszeutfpr.edu.br
}

\begin{abstract}
The World Health Organization (WHO) considers noise pollution a serious environmental problem capable of causing harm to all living beings and because of this, it must be constantly monitored. This article proposes the description of an architecture for the monitoring of noise through the collection of data using smartphones and the visualization of this data in a $3 D$ virtual city. It is possible to implement the proposed solution and it is believed that visualization in a three-dimensional environment will facilitate the analysis and exploitation of the data.
\end{abstract}

Resumo. A Organização Mundial da Saúde (OMS) considera a poluição sonora um grave problema ambiental capaz de causar danos a todos os seres vivos e devido a este fato, deve ser monitorada constantemente. Este artigo propõe a descrição de uma arquitetura para a monitoração de ruídos através da coleta de dados utilizando-se smartphones e a visualização destes dados em uma cidade virtual 3D. É possível implementar a solução proposta através das API's descritas e acredita-se que a visualização em um ambiente tridimensional irá facilitar a análise e exploração dos dados.

\section{Introdução}

Em poucas décadas, grande parte da população mundial estará vivendo em áreas urbanas. As cidades irão crescer em tamanho e complexidade ocasionando uma diversidade de problemas que podem ser difíceis de serem solucionados [Monzon 2015].

Um destes problemas é a poluição sonora, que é altamente prejudicial para o meio ambiente e todos os seres vivos, especialmente os seres humanos. Estudos mostram que nas grandes cidades, $65 \%$ da população estão com a qualidade de vida prejudicada devido aos ruídos intensos e excessivos do ambiente na qual estão inseridas [Bulkin et al. 2015].

A OMS considera a poluição sonora uma ameaça para a saúde pública, pois afeta o sistema nervoso central e cardiovascular, interfere nas atividades diárias das pessoas (em casa, no trabalho, nas escolas e no lazer) reduzindo seu desempenho e afetando seu humor, causando efeitos psicofisiológicos e provocando mudanças de comportamento social [OMS 2011]. Devido aos problemas causados pela poluição sonora, é necessário monitorar a intensidade de ruídos do ambiente para que ações eficazes sejam implementadas [Zappatore et al. 2016].

Diante dos fatos relatados anteriormente, este artigo propõe a descrição de macro processos que irão compor uma arquitetura de software cujo objetivo é monitorar a intensidade sonora de um ambiente urbano. Como resultado é apresentado a ideia de uma 
arquitetura composta por três componentes. O componente 1 descreve uma aplicação para smartphones cuja função é coletar dados referentes a ruídos, o componente 2 descreve um servidor de aplicação para integrar a coleta e visualização de dados e por fim, o componente 3 descreve a criação de um módulo para uma cidade virtual 3D já funcional. O objetivo deste módulo é permitir a visualização dos dados coletados utilizando-se a metáfora de "bolhas sonoras".

Observando-se o fluxo de funcionamento da arquitetura e a descrição das principais API's e métodos que devem ser utilizados, é possível concluir que a proposta deste artigo é viável de ser implementada e atinge o objetivo de monitorar a intensidade sonora de um ambiente urbano.

Acredita-se que a visualização dos ruídos em forma de bolhas sonoras a partir de uma cidade virtual 3D é um diferencial da arquitetura, pois oferece uma maneira intuitiva e eficaz de explorar e compreender estes dados.

\section{Materiais e métodos}

A fim de monitorar a intensidade sonora, este artigo propõe uma arquitetura constituída por três componentes: (1) coletar dados, (2) gerenciar dados e (3) visualizar dados. Esta arquitetura é ilustrada na figura 1.

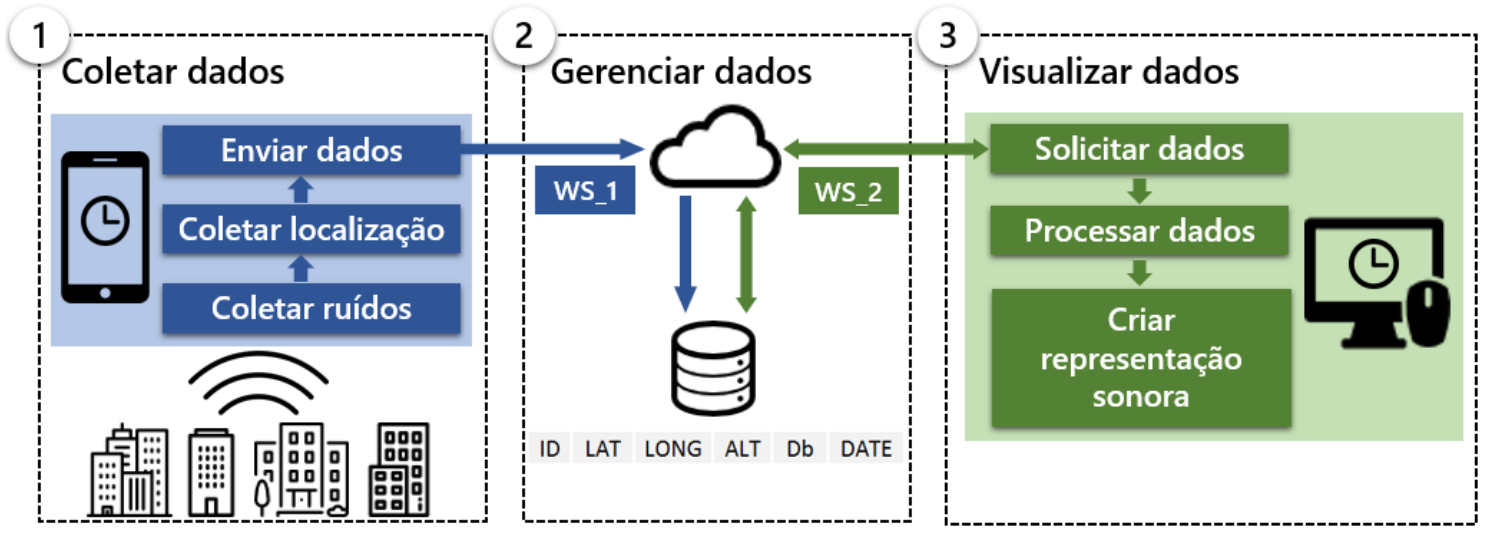

Figura 1. Arquitetura para monitorar a poluição sonora de um ambiente urbano.

O componente 1 é a descrição de uma aplicação para smartphones (neste primeiro momento, apenas para Android) cuja função é coletar dados e enviá-los para o componente 2. Entende-se por dados, a intensidade sonora do ambiente e a geolocalização do smartphone, que será considerada como a posição do ruído. Este componente possui três processos executados em sequência a cada minuto.

- Coletar ruídos: obtém a intensidade sonora com o método getMaxAmplitude() da API MediaRecorder ${ }^{1}$.

- Coletar localização: obtém a geolocalização do smartphone com os métodos getLatitude(), getLongitude() e getAltitude() da API Location².

- Enviar dados: envia os dados coletados para o módulo 2 através do web service WS_1. Deverá ser utilizado as API's HttpClient ${ }^{3}$ e HttpGet ${ }^{4}$.

\footnotetext{
${ }^{1}$ https://developer.android.com/reference/android/media/MediaRecorder.html

${ }^{2}$ https://developer.android.com/reference/android/location/Location.html

${ }^{3} \mathrm{https} / / / \mathrm{developer}$.android.com/reference/org/apache/http/client/HttpClient.html

${ }^{4}$ https://developer.android.com/reference/org/apache/http/client/methods/HttpGet.html
} 
O componente 2 é a descrição de um servidor de aplicação WebLogic ${ }^{5}$ cuja função é disponibilizar dois web services: WS_1 e WS_2. Ambos devem utilizar o formato JSON.

- WS_1: armazena os dados provenientes do componente 1 em uma tabela da base de dados. Esta tabela é composta pelas colunas ID (chave primária), LAT (latitude), LONG (longitute), ALT (altitude), Db (valor em decibéis da intensidade sonora), DATE (data da criação do registro) e basicamente armazena a posição do ruído e sua intensidade.

- WS_2: recupera os dados (apenas os inseridos no último minuto) enviados pelo componente 1 para serem visualizados no componente 3 .

O componente 3 é a descrição de um módulo que deverá ser desenvolvido para a cidade virtual 3D (Curitiba-ViewPort ${ }^{6}$ ). A Curitiba-ViewPort (C-VP) é uma cidade virtual 3D construída com o motor de jogo Unity 3D que representa a cidade de Curitiba/PR e tem o objetivo de ser uma plataforma para desenvolver aplicações com propósitos sérios [Miranda and Stadzisz 2016].

O componente 3 possui três processos executados em sequência a cada minuto.

- Solicitar dados: obtém os dados armazenados no módulo 2 através do web service WS_2. Deverá ser utilizado a API $W W W^{7}$.

- Processar dados: recebe e cria uma lista de dados para ser utilizado no processo a seguir.

- Criar representação sonora: produz a visualização dos ruídos através de bolhas sonoras que devem ser posicionadas de acordo com os valores de latitude, longitude e altitude. Ruídos de baixa intensidade serão representados por tons de verde, média intensidade serão representados por tons de amarelo e tons de vermelho irão representar alta intensidade. Estas bolhas devem ser criadas com o método $O b$ ject.Instantiate ${ }^{8}$. A simulação de como seria esta representação na cidade virtual $3 \mathrm{D}$ pode ser visualizada figura 2 .

\section{Resultados}

Este artigo apresenta uma arquitetura de sistema composta por três componentes para monitorar a intensidade sonora de um ambiente urbano.

- Componente 1: descreve uma aplicação para coletar a intensidade e geolocalização dos ruídos utilizando-se smartphones.

- Componente 2: descreve um servidor de aplicação para integrar a coleta de dados e a visualização dos mesmos através do uso de web services.

- Componente 3: descreve uma estrutura de um módulo para uma cidade virtual 3D com o objetivo de prover a visualização dos ruídos em forma de bolhas sonoras.

\footnotetext{
${ }^{5} \mathrm{http} / / /$ www.oracle.com/technetwork/middleware/weblogic/overview/index-085209.html

${ }^{6} \mathrm{https}: / /$ youtu.be/RPLp4D5N1Ac

${ }^{7}$ https://docs.unity3d.com/ScriptReference/WWW.html

${ }^{8}$ https://docs.unity3d.com/ScriptReference/Object.Instantiate.html
} 


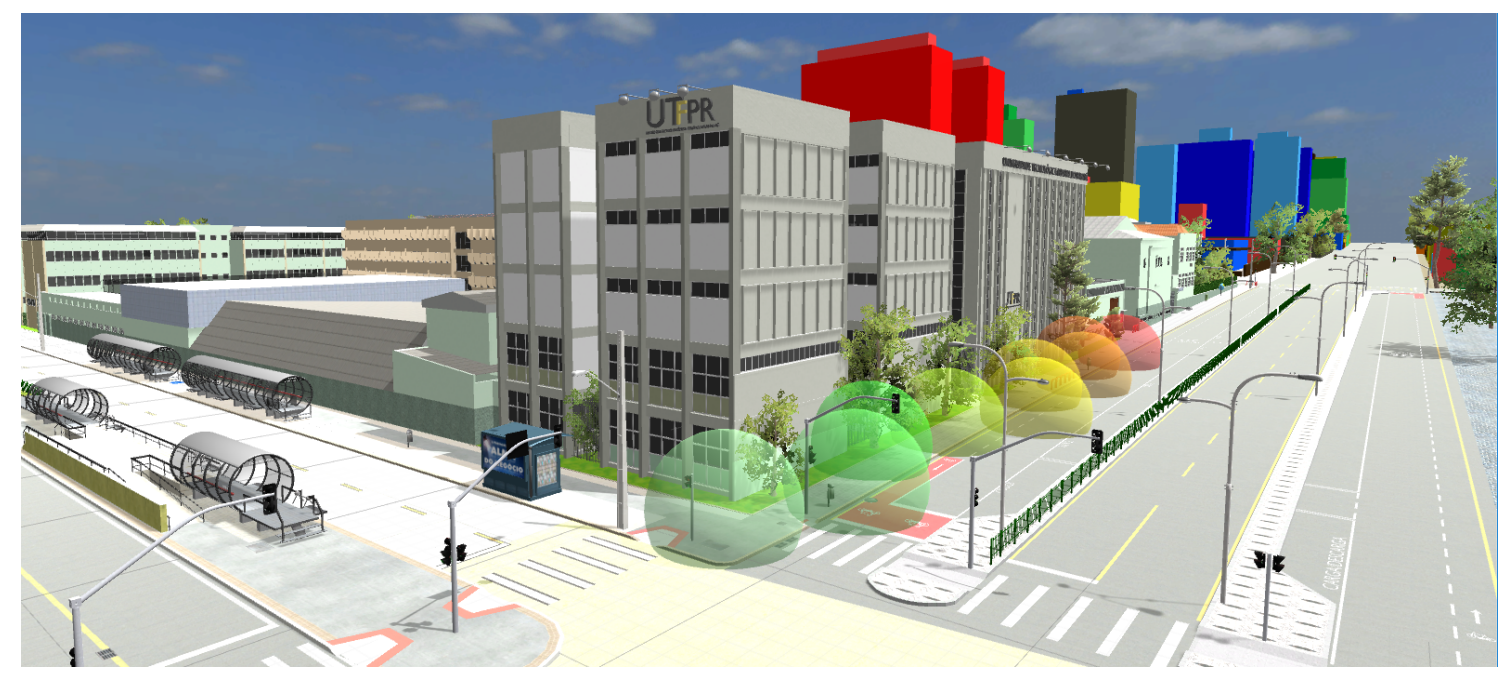

Figura 2. Representação de ruídos na C-VP utilizando-se a metáfora de bolhas sonoras. As posições das bolhas dependem da posição geográfica do smartphone e suas cores estão relacionadas com a intensidade sonora.

\section{Conclusão}

A partir da descrição realizada na seção 2 abordando quais os principais processos envolvidos na arquitetura e como devem ser implementados, é possível concluir que esta arquitetura é totalmente viável de ser implementada e poderá ser utilizada na cidade de Curitiba/PR. Também é possível implementar para outras cidades, mas antes, é necessário expandir a modelagem gráfica da C-VP para englobar outros municípios.

Importante destacar que visualização dos dados em uma cidade virtual 3D facilita a exploração e análise das informações coletadas, podendo tornar-se uma nova forma de compreender diversos dados urbanos.

\section{Referências}

Bulkin, Kalinichenko, and Sal'nikov (2015). About the possibility of the use of dualchamber acoustic screens-resonators to reduce noise. Noise. Theory and Practice, $1(1): 32-41$.

Miranda, F. S. and Stadzisz, P. C. (2016). Curitiba-ViewPort: uma cidade virtual para centralizar aplicações. SLAT JOGOS - $1^{\circ}$ Simpósio Latino-Americano de Jogos, 2(4):4651.

Monzon, A. (2015). Smart Cities Concept and Challenges: Bases for the Assessment of Smart City Projects. 2015 International Conference on Smart Cities and Green ICT Systems, page 11.

OMS (2011). Burden of disease from environmental noise. WHO Regional Office for Europe, 1 edition.

Zappatore, M., Longo, A., and Bochicchio, M. A. (2016). Using mobile crowd sensing for noise monitoring in smart cities. International Multidisciplinary Conference on Computer and Energy Science, SpliTech 2016. 